\title{
Adversidades psicológicas y conceptuales del sordo ante la lectura
}

Christian Israel Huerta Solano, Julio Agustín Varela Barraza, Jairo Alejandro Figueroa González, Laura Gabriela Mariscal de Santiago, Belén Itzel Gómez García

\section{Resumen}

La educación bilingüe fue acogida como forma de alfabetización de las comunidades sordas hasta los años noventa, siendo influida entonces por el paradigma cognitivo, adoptó términos como la conciencia fonológica y la memoria de trabajo. Sin embargo, bajo dichas concepciones, no hay explicación de por qué el sordo continúa sin elevar su nivel lector más allá del cuarto grado de educación elemental, lo que ha permeado en su desarrollo psicológico. Por lo tanto, se considera necesaria la revisión de algunas situaciones presentes en la mayoría de los estudios: 1) la caracterización del sordo; 2) los instrumentos de evaluación de lectura utilizados; 3) las líneas de investigación existentes; 4) el marco teórico utilizado, y 5) la competencia de la Lengua de Señas (LS) de los investigadores. Partiendo de lo anterior, se llega a la conclusión de que es imprescindible usar nuevos paradigmas, como el interconductismo, que permita desarrollar metodologías de enseñanza basadas en las características de los lectores sordos, en un ambiente bilingüe y bicultural. 
Palabras clave: Sordera; lectura; Lengua de Señas; interconductismo.

\section{Introducción}

Los investigadores Andrews, Byrne y Clark (2014) refieren que aunque los primeros trabajos empíricos dedicados a evaluar e intervenir en el nivel lector del sordo fueron los de Rudolf Pintner (Paul, 1998), fue a partir de los años setenta que términos como bilingüismo, manualismo, oralismo, vocabulario, derechos lingüísticos, entre otros, cobraron relevancia para el impulso de metodologías de comunicación que permitían mejorar el nivel lector del sordo, sin embargo, fue hasta 1990, aproximadamente, que se adoptó de forma más o menos homogénea el bilingüismo (señas y lengua escrita) como la forma más adecuada para alfabetizar al sordo (Supalla, 1990).

Precisamente en el período referido (1970-1990) se tiene registro de diversos cambios en la conceptualización de la lectura, principalmente bajo la influencia del paradigma cognitivo, la lingüística y las ciencias sociales (Andrews, Byrne y Clark, 2014), con ello se identificó que la lectura es más que la decodificación y reorganización de las palabras escritas en una página ya que, de acuerdo con la propuesta cognitiva, durante la lectura están implicados procesos cognoscitivos (memoria, atención, percepción) con los que el lector construye significados a partir del texto (Anderson, 1994).

Así, bajo la influencia del paradigma citado, respecto a la conceptualización de la lectura, de manera constante se ha afirmado que el aprendizaje de la lectura en personas sordas es distinto al de las personas oyentes (Hanson, 1989), basándose, sobre todo, en estudios que dedican sus esfuerzos a evaluar el efecto que tiene la conciencia fonológica en la adquisición de vocabulario y mejorar el nivel lector del sordo (Paul, Wang y Williams, 2013), además de otras propuestas que enfatizan el rol de las habilidades de codificación y desarrollo el fonológico aunado a principios semánticos, morfológicos y pragmáticos que parten de principios fundamentalmente oralistas. 
Algunos autores (Luckner, Sebald, Cooney y Muir, 2005, 2006) coinciden en que la lectura es una competencia esencial para el desarrollo psicológico de cualquier individuo, lo que incluye a las personas sordas, cuya situación no debería ser distinta. Sin embargo, en los últimos años, en diversos estudios se reporta que el nivel lector del sordo no sobrepasa el cuarto grado de educación elemental, sin importar que curse grados superiores (ChaII, Jacobs y Baldwin, 1991; Traxler, 2000). Esta situación cobra relevancia no sólo por tratarse de una deficiencia de índole educativa, que aunque ha sido estudiada desde diversas aristas por el paradigma cognitivo, los resultados son similares después de casi 90 años, en relación con los primeros estudios realizados en Estados Unidos (Paul, 1998). Es decir, no se resta mérito alguno a los trabajos realizados, dado que permiten tener un panorama del estado actual de la problemática referida, sino que es cuestionable que no se hayan hecho acciones que vayan más allá de la evaluación y publicación de resultados que, en cierta medida, remiten a cuestionar la base epistemológica y empírica de éstos.

A pesar de los datos referidos, algunos autores coinciden en que, aunque hay diferencias sensoriales referentes a la recepción del sonido en las personas sordas, la situación de los estudiantes sordos no difiere sustancialmente de la de los oyentes al aprender a leer (Adams, 1990; Chall, 1996), considerando que tienen los demás sentidos preservados, aunque usualmente dejan de lado al menos dos aspectos: 1) la cultura del sordo y 2) la forma en que aprenden la lengua oral y escrita, casi siempre a la par de que adquieren la competencia en la Lengua de Señas.

En otras palabras, no basta con realizar evaluaciones e intervenciones para mejorar el nivel lector del sordo que se basen únicamente en el déficit sensorial, sino que dichas investigaciones tendrían que partir del uso de los vocablos, acciones, costumbres y modos de vida de aquellos sordos que reciben una educación bilingüe (LSM-Lengua escrita) y bicultural (cultura sorda-oyente), dado que de otra forma se estarían sesgando tanto los resultados como el propósito fundamental de éstos, lo que en buena parte podría explicar el estatus actual del problema. Por lo tanto, valdría la pena analizar los elementos aludidos en la mayoría de los estudios en los que se evalúa o procura 
mejorar el nivel lector del sordo, entendiendo que actualmente la principal contribución estaría en el análisis del contexto y la forma en que han sido elaborados ciertos constructos en torno al sordo y la manera de lograr la competencia lectura, en lugar de elaborar más escalas fundamentadas sobre una cuestionable validez de constructo.

Partiendo de la premisa anterior, consideramos relevante la revisión de algunos elementos que podrían aportar cierta claridad al tratar de interpretar el lánguido avance en la mejora del nivel lector del sordo, éstos son: 1) la caracterización del sordo; 2) los instrumentos de evaluación de lectura utilizados con estudiantes sordos; 3) las diversas líneas de investigación existentes; 4) el marco teórico utilizado, y 5) la competencia de la Lengua de Señas (LS) de los investigadores.

\section{Caracterización del sordo}

En primer lugar, consideramos relevante hacer una revisión de la caracterización que se tiene del sordo, limitada al nivel de pérdida auditiva y de acceso a un código fonológico, por lo que se discurreo que a partir de ésta es posible comprender, al menos en parte, algunos aspectos que hasta el momento han sido considerados en algunas investigaciones.

Autores como Pintner y Paterson (1927, citado en Paul 1998), Herrera (2005) y Schimmel, Edwards y Prickett (1999) afirman que para poder llevar a cabo el aprendizaje de la lectura es necesario que el sordo tenga acceso a un código fonológico. Ante esta premisa, se observan dos implicaciones subyacentes relevantes: por un lado, la homogenización de los sordos e hipoacúsicos, lo cual implicaría que los métodos de enseñanza de la lectura serían similares para todos, incluyendo a sus coetáneos oyentes, y con esto se descartaría la necesidad de desarrollar un método de enseñanza de la lectura exclusivo para la población sorda; por el otro, que la sordera no tendría que ser clasificada más allá del grado de pérdida auditiva, considerando la capacidad del estudiante para tener acceso al lenguaje oral (sonoro), por lo tanto, desde esta postura se entiende que a aquellos sordos les resultara imposible tener acceso a dicho 
código fonológico, y también sería inútil dedicar esfuerzos para que éstos lograran al menos un nivel ínfimo de competencia lectora. Esta situación parece sumamente limitada, aunque existe un gran número de estudios que parten de dicha clasificación para sustentarlos.

Lo anterior permite hipotetizar por qué desde Pintner, en 1927, pasando por las investigaciones realizadas por el National Reading Panel (2000) y otras como las de Schirmer y Williams (2003), continúan dedicando sus esfuerzos a la evaluación de las habilidades fonológicas de los sordos en lugar de llevar a cabo programas de intervención adecuados para el desarrollo de sus competencias lectoras, mismos que, como ya se indicó, carecen de un contexto bilingüe y bicultural en el que los estudios pudieran ser realizados en consonancia con las necesidades reales del lector sordo.

En relación con lo anterior, vale la pena indicar que algunos investigadores (DeVilliers y Pomerantz, 1992) incluyen, de forma indiscriminada, participantes con diverso grado de pérdida auditiva, cuando, por el contrario, en muchos de estos casos resulta obvio que aquellos con un grado menor a los $40 \mathrm{~dB}$ (hipoacúsicos) obtienen mayores beneficios que aquellos que tienen una pérdida auditiva superior a los 70 u 80 dB (sordera severa o profunda), lo que determina, en muchas ocasiones, que los sordos que no son capaces de desarrollar habilidades lectoras por medio del oralismo o medios sonoros, sean etiquetados como incompetentes o, incluso, con un nivel cognitivo inferior al de sus coetáneos oyentes, tal como lo indica Pintner (Paul, 1998).

Por consiguiente, consideramos importante hacer una adecuada caracterización del sordo respecto a su nivel de pérdida auditiva: uso o ausencia de aparatos auditivos auxiliares, su acceso o no al entrenamiento oralista. Por otra parte, hay que considerar la enseñanza de la lectura a partir de referentes no fonológicos, esto a través del apoyo de elementos visuales, cinestésicos y quinestésicos, realizando adaptaciones no sólo para evaluar el nivel lector, auditivo y de vocabulario de los estudiantes, sino también para implementar un método acorde con sus necesidades educativas, lo que incluye caracterizar al participante sordo en su contexto natural, cultural, "bilingüe" (Lengua 
de Señas Mexicana y castellano escrito) y escolar, sin compararlo con el oyente; además de enseñarle la "segunda" lengua a partir del conocimiento de su lengua nativa (Lengua de Señas).

Así, Sánchez López y Rodríguez (1997) argumentan que el bilingüismo en la cultura sorda puede ser clasificado según la identidad cultural, de la que se derivan el bilingüismo cultural y el bilingüismo monocultural. En el caso del sordo bilingüe bicultural, éste convive con los miembros de la comunidad sorda, así como con las personas de la sociedad oyente, valorando y aceptando ambas culturas, sin referir superioridad o inferioridad con respecto a la comunidad sorda u oyente. Por otro lado, el sordo bilingüe monocultural sólo se apropia de la cultura de uno u otro grupo, aunque puede conocer y utilizar ambas lenguas. Siguiendo este modelo, no bastaría con evaluar el nivel lector del sordo partiendo de los estándares de la cultura oyente oralista dominante, sino que es necesario tomar en cuenta tanto el aporte de la Lengua de Señas (LS) como de la cultura en que el LS se usa para poder llevar a cabo una intervención acorde con la realidad y con la necesidad psicológica, además de lingüística, de los participantes sordos.

\section{Instrumentos de evaluación lectora para sordos}

En muchos estudios revisados, sobre todo los relacionados con la evaluación de habilidades lectoras de estudiantes sordos, entre éstos los del Center for Assessment and Demographic Studies (CADS) de la Universidad de Gallaudet y los del Scholarship Aptitude Test (SAT) en sus diversas versiones (1969-2010), se lleva a cabo la adaptación de instrumentos diseñados para población oyente, lo cual implica que el estudiante sordo sea evaluado a partir de estándares que pueden diferir en gran medida de sus características sensoriales y de su contexto habitual, además de la frecuencia y uso del vocabulario en la elaboración de frases, tomando en cuenta que la sintaxis de la Lengua de Señas difiere del inglés, castellano, portugués, etcétera, lo cual repercute en los resultados respecto al nivel lector de los participantes sordos, además de que la norma de comparación sigue siendo la población oyente. 
En este sentido, muchas de las adaptaciones realizadas para evaluar el nivel lector y de vocabulario del sordo son llevadas a cabo por oyentes (LaSasso y Davey, 1987), además de que éstos conocen poco o nada de la Lengua de Señas, así como de la cultura sorda. Por lo tanto, más allá de ser una adaptación, resulta ser una transcripción de frases que distan del uso de elementos relevantes y concordantes con la lengua primaria de los participantes, omitiendo, según sus apreciaciones, aquellas cuestiones que, dada la comparación con los oyentes, en un sordo resultarían irrelevantes diagnosticar, por ejemplo, el uso de plurales, conectivos y conjugaciones.

Dada la incongruencia respecto a los estándares de evaluación indicados, es importante elaborar instrumentos que permitan realizar una adecuada evaluación de la competencia lectora del estudiante sordo (Huerta-Solano, 2014), no sólo a partir de los programas y planes ofrecidos por la Secretaría de Educación Pública, sino corroborando el uso y permanencia de la Lengua de Señas. Es decir, se requiere la familiaridad, frecuencia y uso de los vocablos en Lengua de Señas Mexicana en sus contextos naturales para, posteriormente, lograr su equiparación respecto al grado escolar y el nivel lector esperado de acuerdo a la edad de los participantes (Varela, Huerta-Solano, Nava y Ortega, 2014), lo que muy probablemente garantizará la prevalencia de la lengua primaria (Berko, 1958; Cooper, 1967 y Raffin, 1976; en Paul, 1998), además de la evaluación a partir del contexto y uso real de los vocablos que son presentados a los estudiantes, descartando el uso de evaluaciones adaptadas y el contexto y necesidades específicas de los participantes, lo cual delimita los elementos evaluados (vocabulario y sintaxis en castellano) a partir de sus características.

\section{Líneas de investigación en la enseñanza de la lectura a los participantes sordos}

A pesar de que existen diversas vertientes tradicionales de investigación (ilustraciones, vocablos aislados, LS, sinónimos, etcétera) respecto a la enseñanza de la lectura en participantes sordos, Paul (2003) destaca que casi de forma homogénea se 
trata de indagar acerca del nivel de vocabulario que tienen los participantes sordos, por lo que usualmente se pretenden corroborar tres líneas de investigación: 1) la amplitud y dominio de vocabulario; 2) la relación existente entre el conocimiento de las palabras y la comprensión lectora, y 3) la evaluación de las habilidades de los estudiantes para derivar el significado de las palabras a partir de pistas del contexto. Sin embargo, se aclara que actualmente en las líneas de investigación referidas se observan algunas dificultades en su fundamentación y desarrollo (LaSasso y Davey, 1987; Paul y Gustafson, 1991), tal como se especifica a continuación.

Respecto a la amplitud y dominio de vocabulario, algunos investigadores (Balow, Fulton y Peploe, citados en Paul, 1998) no ofrecen una descripción exacta tanto del nivel de dominio como del número de vocablos que el participante sordo debe conocer basándose en referentes específicos (edad, contexto, nivel sordera, escolaridad, etcétera), y en caso de proporcionarla (Mies, 1992), el referente continúa siendo únicamente el conocimiento de vocablos escritos y no el dominio conceptual, uso sintáctico, ni su relación con la Lengua de Señas "nativa" del participante.

Por otra parte, algunos estudios de conocimiento de palabras y comprensión lectora parten de la premisa de que al enseñar vocablos el participante también sabrá hacer uso sintáctico y pragmático de éstos durante la lectura. Sin embargo, tal como lo menciona Herrera (2005) en el estudio de Gaines, Mandler y Bryant (1981), los participantes sordos son capaces de aprender un número considerable de palabras, sin embargo, al evaluar su comprensión de diversos textos, los participantes realizan interpretaciones aisladas y carentes de sentido, basándose únicamente en aquellas que les resultan familiares, las que distan de la integración y comprensión de la lectura.

En relación con los estudios dedicados a derivar el significado de las palabras a partir de pistas del contexto, entre éstos los de MacGinitie (1969, citado en Paul 1998), DeVilliers y Pomeratz (1992), Schimmel, Edwards y Prickett (1999), es posible constatar algunos errores derivados de las dos líneas anteriores. Al ofrecer pistas de los vocablos a los participantes, se parte del 
supuesto de que la mayoría conoce el significado de las palabras en su totalidad, aunado a que son escritas con base en la sintaxis de la lengua pero en su forma oral. Además, en muchos casos se hipotetiza la correspondencia del lenguaje oral y escrito en relación con la Lengua de Señas al hacer uso de conectivos, verbos conjugados y preposiciones, lo cual resulta incorrecto, ya que con los resultados de diversos estudios, entre ellos los de Lederberg y Everhart (2000), además de Dimling (2010), se ha corroborado que un alto porcentaje de sordos desconoce el uso sintáctico y pragmático de dichos vocablos en la conformación y lectura de un texto en su forma escrita. Por lo cual resultan evidentes los sesgos metodológicos en muchos de los estudios dedicados a esta línea de investigación.

\section{Marco teórico de la investigación con sordos}

Como se indicó al inicio, la mayor parte de las líneas de investigación referidas se han llevado a cabo bajo el paradigma cognitivo, por lo que investigadores como Paul (1998) refieren que el logro de un alto nivel lector en los participantes sordos ha llevado a muchos autores a argumentar que la lectura es una actividad cognitiva o de razonamiento. Por ejemplo, Aceti y Wang (2010) sugieren, a partir de un estudio realizado por Paul en 1997, la necesidad de la activación de aprendizajes y conceptos previos respecto al uso de las palabras; otros como Smith, Hynd y Valeri-Gold en 1990 (citados en Paul, 1998) indican la necesidad de hacer predicciones, usar imágenes, relatar el conocimiento previo respecto al uso de la palabra leída, verbalizar las situaciones confusas y emplear la autocorrección.

Sin embargo, dichos estudios aún presentan elementos aislados del problema, entre éstos, la necesidad de verbalización u oralización, la omisión de la Lengua de Señas como base para el aprendizaje de vocablos y su integración en textos escritos, la exclusión de instrucciones en diversas modalidades (escrita, labiofacial, Lengua de Señas), además de que se parte del supuesto de que los participantes conocen tanto el mismo número de conceptos como sus relaciones funcionales y semánticas, por lo que consideramos que las objeciones referidas se 
derivan del paradigma que se tiene como base, el cual podría generar resultados favorables en casos de participantes hipoacúsicos (Quigley, McAnally, King y Rose, 1991; Quigley, McAnally, Rose, Payne y Paul, 2003).

Si se revisa lo anterior, el uso de los referidos constructos hace evidente la problemática inherente a la base epistemológica el paradigma cognitivo, considerando que no hay otro medio para acceder a la lectura más que el código fonológico y la comprensión de los vocablos, gramática y sintaxis escrita de no ser de forma oral o escrita, olvidando que la lengua que usa el sordo tiene elementos visogestuales, no transferibles a la escritura. Por lo tanto, y como ya se ha constatado en diversos programas de intervención, es cuestionable que se continúe haciendo uso del paradigma cognitivo cuando el aprendizaje de participantes con sordera severa y profunda es predominantemente práctico y de referencia inmediata, además de ser visogestual. Es decir, no serían necesarios los elementos "metacognitivos" que se aluden en el cognoscitivismo, pues tienen poca utilidad para tratar de explicar la forma en que el sordo comprende la lengua escrita u oral, considerando que esto sería mejor explicado a partir de las relaciones funcionales que proporciona el lenguaje y no haciendo uso de elementos extracorpóreos para tratar de explicar las inconsistencias derivadas de la condición sensorial y experiencial del sordo, además de las diferencias entre la lengua escrita y la Lengua de Señas.

A partir de lo anterior, es necesario contar con otro paradigma de referencia, en este caso el de Kuhn (1962/2004) quien refiere que en los periodos normales de la ciencia, el científico investiga determinada área del conocimiento con problemas plenamente definidos, los que de no ser resueltos, como actualmente sucede con el nivel lector del sordo bajo el paradigma cognitivo, usualmente se alude al modelo, aunque no se toma en cuenta que en el corpus existen anomalías y omisiones que con el tiempo devienen en crisis, que de no resolverse, sobre todo si se refiere a necesidades o demandas sociales apremiantes, podrían llevar al rechazo del paradigma vigente.

Consecuentemente, el modelo cognitivo actual de enseñanza y evaluación de la lectura para el sordo, considerando la 
necesidad apremiante de elevar los niveles de sus participantes, ha dejado de lado la Lengua de Señas, la cultura y el contexto de interacción de los estudiantes sordos para lograr este fin, lo que se hace mucho más evidente al empatarlo con las dificultades inherentes al propio corpus conceptual del paradigma usado, sobre todo al identificar y adecuar el uso de constructos tales como la conciencia fonológica, acceso a un código fonológico, nivel cognitivo inferior, dificultades en la memoria de procesamiento, espacios mentales, entre otros (Cruz-Aldrete 2014).

En la actualidad, si bien es cierto que no hay suficientes estudios en lengua escrita castellana mexicana, aunada al uso de la LSM, y cuyos participantes sean sordos mexicanos, sí se reconoce el trabajo que otros paradigmas en psicología hacen para procurar resolver las inconsistencias teóricas y empíricas, partiendo de su inconmensurabilidad, que existen en la enseñanza y evaluación del nivel lector del sordo. Recientemente, la propuesta teórica interconductual (Kantor y Smith, 1975, 2015; Ribes y López, 1985; Varela, 2008) ha provisto contribuciones en lo que respecta a la elaboración de instrumentos, evaluación e intervención en la enseñanza de vocablos y la mejora del nivel lector que pueden beneficiar a estudiantes sordos.

Aludiendo a la primicia de las investigaciones de corte interconductual en la evaluación e intervención para mejorar el nivel lector del sordo, aún es pronto generalizar los resultados obtenidos en éstas, sin embargo, se destaca que, a diferencia de la propuesta cognitiva actual, aquélla permite considerar otras aristas usualmente omitidas o tergiversadas: se destaca la importancia de la interacción que tiene la persona sorda con su contexto para su desarrollo psicológico; se asume la relevancia de la enseñanza bilingüe y bicultural; a diferencia de otras propuestas, se dota de convencionalidad a la Lengua de Señas y no sólo se la considera un "lenguaje" de signos, sino una lengua que permite sustituir contingencias y lograr el desligamiento funcional extrasituacional de cosas y hechos concretos; se hace factible el análisis de los hechos a partir de una teoría psicológica, dejando de lado la transaccionalidad de términos multívocos provenientes de otras disciplinas y teorías lingüísticas, tales como la cibernética y la informática; se parte de las caracterís- 
ticas de los participantes sordos, ya que éstas no son una limitante para su desarrollo psicológico, dado que con el paradigma interconductual el individuo puede lograrlo en tanto esté vivo; y, por último, se otorga una definición clara de la competencia lectora en el sordo, sin aludir a entidades transcorpóreas (espacios mentales, consciencia fonológica, memoria de trabajo), sino basada en las relaciones funcionales que tiene el lenguaje, a saber, "la aptitud que tiene el lector (referido) para establecer contacto funcional con lo que se lee (referente), tal cual fuera él mismo el escritor (referidor); dado que de otra manera no sería posible el acto lingüístico, puesto que no cumpliría con el criterio de que la interacción sea biestimulacional (Huerta-Solano, Varela, Figueroa, González y Flores, 2016)".

Como ejemplo de lo anterior, en un estudio reciente que tiene como base la propuesta interconductual, Varela, Huerta-Solano, Nava y Ortega (2014) comprobaron la efectividad de enseñar vocablos aislados en castellano y posteriormente incorporarlos a frases con cinco o seis palabras auxiliándose de la Lengua de Señas, y además de que parten de las características de los participantes sordos (nivel de uso de LS, nivel de pérdida auditiva, biculturalismo, monoculturalismo, etcétera). Por su parte, Tello-Miranda, Varela y Palos-Toscano (en dictamen) realizaron una réplica del estudio mencionado en una muestra similiar de sordos de la que obtuvieron resultados favorables.

Más recientemente, Huerta-Solano, Varela, Figueroa, González y Flores (2016) Ilevaron a cabo una evaluación del nivel lector del sordo en una población mexicana que hacía uso de la LSM, partiendo de la propuesta conductual. Se destaca que aunque se corroboraron los datos respecto al nivel lector del sordo obtenidos bajo el paradigma cognitivo, se logró evaluar a partir de estándares acordes con el contexto, Lengua de Señas y cultura mexicana del sordo, lo cual evidenció la pertinencia del paradigma interconductual para lograr conceptualizar adecuadamente la forma en que el sordo aprende y usa el vocabulario mediante ilustraciones (modo observación), vocablos escritos (modo lectura) y uso de la Lengua de Señas (actuaje), los cuales son teóricamente sustentables bajo el concepto de interconducta lingüística (Kantor y Smith, 1975, 2015). 
Como se observa, actualmente se cuenta con poca investigación en lo que respecta al uso del paradigma interconductual en el área, aunque se destaca que las contribuciones que se han hecho se fundamentan en el respeto a la cultura del Sordo y la Lengua de Señas, teniendo en cuenta una fundamentación empírica y teórica basada en la realidad biológica y psicológica del Sordo, lo que puede en un futuro disminuir los sesgos y la búsqueda de elementos fonológicos para evaluar a los Sordos a partir de su contexto habitual.

\section{Competencia de la Lengua de Señas}

Por otra parte, es necesario considerar la competencia del experimentador en el uso de la Lengua de Señas que, aunque en algunos experimentos pareciera tener una relevancia menor, se puede corroborar su influencia al reportar los resultados. Así, en una gran parte de los experimentos realizados con participantes sordos, independientemente de la línea de investigación (Dimling, 2010; Krakow y Hanson, 1985), algunos autores refieren en su metodología el entrenamiento de terceros por parte de expertos en la Lengua de Señas (maestros, pedagogos, psicólogos) para que lleven a cabo el experimento, siendo entonces los observadores tanto del desempeño de los participantes como de los administradores (intérpretes) del estudio.

Debido a lo anterior, se considera relevante referir algunos de los sesgos metodológicos que pueden ocasionarse. Por un lado, al entrenar a terceros, a pesar de ser expertos en la LS, no se garantiza que las instrucciones sean dadas de acuerdo con las necesidades del estudio, ni bajo las condiciones previstas por el investigador, ya que se considera que éstos sólo fungen como intérpretes y no como especialistas en el área de estudio. Además, la falta de conocimiento de la LS por parte del investigador lo mantiene al margen de los supuestos dados por la literatura generada hasta el momento, junto con una limitada inclusión en la cultura del sordo, aunque quizás el elemento más relevante sea la falta de conocimiento acerca de la morfología, topología y significación contextual de muchas de las señas; y es que se debe tomar en cuenta que, al igual que las lenguas 
dominantes, la LS depende del contexto, praxis y uso sintáctico. Por ejemplo, el intérprete podría referir que tal o cual resultado es correcto, pero el investigador no tendría los medios para constatar la veracidad del hecho, es decir, resulta imposible, al menos in situ, constatar la veracidad de la información que se está registrando.

Por lo tanto, se considera importante documentar la experiencia en Lengua de Señas tanto del experimentador como de los colaboradores, además de verificar la equivalencia entre las señas y los vocablos escritos usados en los estudios. También, se añade la relevancia de interpretar y verificar tanto la compresión de las instrucciones como de cada uno de los procedimientos, garantizando así el uso adecuado de la Lengua de Señas, además de su administración bajo las consideraciones axiológicas y legales a nivel contextual y a nivel cultural.

\section{Discusión}

Ante los argumentos expuestos, consideramos pertinente una revisión de los elementos teóricos y empíricos bajo los que se llevan a cabo las evaluaciones e intervenciones respecto a la mejora del nivel lector en estudiantes sordos, principalmente enfatizar en el uso de diversos estándares (dactilología, discriminación de imágenes, seña), partiendo de un contexto bicultural y bilingüe, y promoviendo de manera enfática el aumento del vocabulario, aunado a los elementos sintácticos que componen una frase en castellano y en Lengua de Señas, además de su uso y significación en un texto habitual. Por ejemplo, algunos programas como el Fairview Reading Program (Schimmel et al., 1999), entre otros, llevan a cabo la intervención a través de elementos aislados y sin asociación alguna, reportando resultados favorables en el postest, sin embargo, se ha comprobado que en las evaluaciones de seguimiento muchos de los participantes de dichos estudios (Nagy y Scott, 2000; Carver, 1994; Chall, Jacobs y Baldwin, 1990), al evaluar su comprensión lectora, siguen sin comprender entre 90 y $95 \%$ de los vocablos que integran un texto. De ahí la importancia de no sólo entrenar el vocablo en su forma escrita (Flaherty y Moran, 2004), sino también incluir refe- 
rentes como ilustraciones, objetos, acciones y sus equivalentes en Lengua de Señas (dactilología y seña).

Aunque el uso de la Lengua de Señas, ilustraciones y vocablos de forma asociada pueden ser la base para la enseñanza de vocablos en sus diferentes modalidades, no es del todo la solución si se utiliza de manera inadecuada, ya que en muchos de los experimentos únicamente se reportan resultados aislados respecto al uso de la Lengua de Señas (Geraci, Gozzi, Papagno y Cecchetto, 2008; Krakow y Hanson, 1985), ilustraciones (Bebko y McKinnon, 1990) y dactilología (Krakow y Hanson, 1985), por lo que dicha situación resulta inadecuada ya que es imposible hacer una comparación conjunta de los resultados obtenidos, dado que en los experimentos se consideraron tres modos no relacionados para determinar el conocimiento del vocablo, los que entre sí mismos son incomparables partiendo de su modalidad, además de que en muchos casos un modo puede resultar predominante sobre otro, lo que depende de la historia de aprendizaje del sujeto.

En un sentido similar, los resultados obtenidos en muchos estudios que hacen uso de la dactilología en la enseñanza de vocablos parecen ser favorables, pero al momento de llevar a cabo la evaluación de la comprensión lectora de los participantes ésta continua sin referir mejora alguna (Krakow y Hanson, 1985), tomando en cuenta que los participantes únicamente repiten los vocablos presentes en el texto impreso, pero desconocen su integración y sentido en la conformación de la palabra (amable-amablemente-amabilidad) y enunciados, incluso se ha podido constatar que algunos de los participantes utilizan la dactilología como recurso primordial al desconocer la palabra o su significado, siendo más evidente en la conjugación de verbos (Huerta-Solano, 2014). Para esto, puede consultarse a Varela, Huerta-Solano, Nava y Ortega (2014), quienes comprobaron que cuando al participante sordo se le presenta, por ejemplo, el verbo comer, éste puede signarlo, seleccionar la imagen y deletrearlo (dactilología) sin dificultad alguna, pero al pedirle que conjugue el verbo (comeremos, comerán), el estudiante recurre al uso de la dactilología, indicando desconocer el vocablo, es decir, debe guiarse por la conformación de letra por letra de la 
palabra y no por su significado, uso o signo en Lengua de Señas Mexicana, lo que alude a los elementos revisados, entre éstos, los instrumentos de evaluación usados y la forma en que son incluidos los modos de forma conjunta o aislada.

Por otro lado, algunos estudios dedicados a la enseñanza de vocablos y que parten del uso de la Lengua de Señas, no toman en consideración su sintaxis, la cual difiere considerablemente de la lengua oral y escrita, elemento considerado en la caracterización actual del sordo, aunque es posible corroborar que los participantes tienen un aumento considerable en el número de signos y vocablos aprendidos en su forma escrita, ya que al solicitarles que lean y signen un texto con las palabras aprendidas durante el entrenamiento, los participantes signan las palabras aprendidas o conocidas por ellos, pero omiten aquellas que en la Lengua de Señas carecen de relación funcional (adverbios, conjugaciones, conectivos, pronombres), hipotetizando que esto se debe a la descontextualización de los elementos usados, por lo que los participantes interpretan el texto predominantemente a partir de sustantivos, adjetivos y verbos en infinitivo. Es decir, se incrementa la competencia del participante en la Lengua de Señas, pero se omite su relación con la sintaxis y pragmática de la lengua escrita (Huerta-Solano, 2014), situación usualmente inherente al paradigma usado.

Lo anterior tiene graves repercusiones en la competencia lectora del participante, ya que en lugar de utilizar la Lengua de Señas como recurso para que el sordo incremente su competencia lectora de la lengua escrita, se da por entendido que éste sólo la necesita como medio de interacción de referencia inmediata (presencia del interlocutor), además se le excluye del aprendizaje de los componentes gramaticales que componen una oración en su forma escrita, dado que no son necesarios en la Lengua de Señas, dispensándole también de referirse a él mismo y a otros en diferentes tiempos verbales, en diferentes circunstancias y relaciones causales, cuando estás son indispensables para el desarrollo del pensamiento abstracto (Dimling, 2010). En otras palabras, se limita el desarrollo psicológico del estudiante sordo, lo que probablemente puede estar relacionado con los instrumentos de evaluación usados, además de la falta 
de conocimiento de los experimentadores tanto de la Lengua de Señas como de la realidad educativa y social del sordo.

\section{Conclusión}

Tomando en cuenta las adversidades que tiene el sordo al procurar el aprendizaje de la lectura, se observa que en las diversas investigaciones llevadas bajo los supuestos y las metodologías que actualmente se consideran predominantes, se cae en un círculo vicioso, ya que el estudiante sordo aprende vocablos aislados, necesarios sólo para su interacción mediante la Lengua de Señas Mexicana con otra persona, pero no se le apoya a ser competente para interactuar con textos que requieren necesariamente del aprendizaje de conectivos, palabras polisémicas, sintaxis, lenguaje figurado y conjugación verbal. por lo que sea cual fuere la teoría en la que se respalde la investigación, Por lo tanto, consideramos que se deben adecuar sus procedimientos para que las personas sordas sean capaces de leer cualquier texto, lo que está relacionado con la revisión de los postulados teóricos, empíricos y deontológicos bajo los que se llevan a cabo las investigaciones

Por ejemplo, Minskoff (2005) refiere que durante las primeras etapas escolares los estudiantes sordos y oyentes adquieren la mayor parte de su vocabulario a partir de referentes concretos (mesa, lápiz, banca, plato, vaso), esperando que a partir del tercero o cuarto grados ambos lean y comprendan la mayor parte de los vocablos presentes en su ambiente habitual. Sin embargo, a partir del quinto y sexto grados van requiriendo la comprensión del lenguaje (Minskoff, 2005) por medio de las relaciones del mismo lenguaje en cualesquiera de sus formas, por lo que se requiere de la comprensión de conceptos y significados sin referentes concretos (relatividad, arbitrario, deductivo, inductivo), situación usualmente olvidada, sobre todo por el uso excesivo de elementos orales, como forma predominante en la enseñanza de lectura del sordo.

Consecuentemente, mientras los estudiantes oyentes escuchan y hacen uso de muchos de los elementos gramaticales para la formación de nuevos conceptos y la generación de diversos significados, los sordos continúan haciendo uso de los referentes inmediatos y concretos como se hace en grados de escolariza- 
ción inferiores, por lo que con ello se argumenta que el estudiante sordo realmente lee un pequeño porcentaje de los vocablos presentes en un texto, limitando la comprensión, interpretación y transformación del mismo, siendo probablemente ésta la razón primordial de la incompetencia lectora de muchos de ellos, aunque se observa que, de tomar en cuenta algunos de los elementos referidos en este trabajo, se procuraría relacionar la práctica actual con la verdadera necesidad que tiene el sordo de adquirir mayores niveles de competencia lectora.

Vale la pena referir que, aunque en una buena cantidad de estudios las respuestas dactilológicas son consideradas como correctas, esto se asume como un sesgo en los resultados, lo que puede adjudicarse al desconocimiento, incluso mínimo, que tienen muchos de los investigadores de la Lengua de Señas, ya que al discurrir que el deletreo de la palabra equivale a conocerla, se da por hecho de que el participante también sabe su significado y que es capaz de diferenciarla y hacer uso discriminado de ésta, lo que equivaldría a decir que el niño oyente sabe leer con sólo repetir las consonantes o vocales.

Por lo tanto, enfatizamos en la necesidad de aclarar que lo que realmente se reporta en estos estudios no es el nivel de conocimiento de vocabulario de los participantes, sino su capacidad para deletrear vocablos, argumento constante en los estudios oralistas de corte cognitivo. En otras palabras, indicar las letras que componen una palabra no sustenta bajo ningún argumento saber hacer uso de la misma, y esto sería similar al hecho de que una persona ante la palabra quintería por deletrearla supiera qué significa. Eso probaría que sabe deletrear, pero no el significado del término, o que por enseñar una seña a la persona normo-oyente ésta supiera qué significa y cómo usarla en una charla con una persona sorda.

Finalmente, coincidimos con autores como Dimling (2010) y Kuntze (1998), quienes sustentan la necesidad de exponer la Lengua de Señas al sordo, pero aunada a diferentes formas de interacción para lograr su aprendizaje (visual, quinestésica, cenestésica, sensorial), tal como se ya argumentó, considerando que esto propiciaría el aumento de las habilidades lectoras, como el desarrollo psicológico de los participantes sordos, ga- 
rantizando, además, su acceso e inclusión en diversas modalidades del lenguaje a partir del desarrollo adecuado de la lengua primaria (LS) para el desarrollo posterior de la lengua escrita.

\section{Conflicto de interés}

La elaboración de este manuscrito tiene como única finalidad dar a conocer la situación actual de la educación del sordo, sobre todo en lo que respecta al tema de la lectura, además de hacer explícita la propuesta interconductual como alternativa a los bastos estudios realizados bajo la propuesta cognitiva.

\section{Financiamiento}

Para la elaboración del presente escrito se contó con el apoyo económico del Consejo Nacional de Ciencia y Tecnología (Conacyt), como parte de su programa de becas para estudios de posgrado.

\section{Referencias}

Aceti, K. E. y Wang, Y. (2010). The Teaching and Learning of Multimeaning Word with Metacognitively Based Curriculum. American Annals of the deaf, 155: 118-123.

Adams, M. J. (1990). Beginning to Read: Thinking and Learning about Print. Cambridge, MA: MIT Press.

Anderson, R. C. (1994). The Future of Reading Research (Technical Report No. 600). Champaign: Center for the Study of Reading, University of Illinois.

Andrew, J. F., Byrne, A. y Clark, M. D. (2015). Deaf Scholar on Reading: A Historical Review of 40 Years of Dissertation Research (1973-2013): Implications for Research and Practice. American Annals of the Deaf, 159(5): 393-418.

Bebko, J. M., y McKinnon, E. (1990). The Language Experience of Deaf Children: Its Relation to Spontaneous Rehearsal in a Memory Task. Child Development, 61: 1744-1752.

Carver, R. P. (1994). Percentage of Unknown Words in Text as a Function of the Relative Difficulty of the Text: Implications for Instruction. Journal of Reading Behavior, 26: 413.

Chall, J. S. (1996). Stages of Reading Development. (2nd ed.). 
Fort Worth: Harcourt Brace Jovanovic College Publishers.

Chall, J. S., Jacobs, V. A. y Baldwin, L. E. (1990). The Reading Crisis: Why Poor Children Fall Behind. Cambridge, MA: Harvard University Press.

DeVilliers, P. y Pomerantz, S. (1992). Hearing-impaired Students Learning New Wordsfrom Written Context. Applied Psycholinguistics, 13: 409-431.

Dimling, L. M. (2010). Conceptually Based Vocabulary Intervention: Second Graders' Development of Vocabulary Words. American Annals of the Deaf, 155: 425-448.

Flaherty, M. y Moran, A. (2004). Deaf Signers who know Japanese Remember Words and Numbers more Effectively than Deaf Signers who know English. American Annals of the Deaf, 149: 39-45.

Geraci, C., Gozzi, M., Papagno, C. y Cecchetto, C. (2008). How Grammar can Cope Withlimited Short-term Memory: Simultaneity and Seriality in Sign Languages. Cognition, 106, 780-804. doi:10.1016/j.cognition.2007.04.014

Hanson, V. (1989). Phonology and Reading: Evidence from Profoundly Deaf Readers. In D. Shankweiler y I. Liberman (Eds.), Phonology and Reading Disability: Solving the Reading Puzzle, pp. 69-89. Ann Arbor: University of Michigan Press.

Herrera, V. (2005). Adquisición temprana de lenguaje de signos y dactilología. Revista Psicopedagógica, 77-78(13): 2-10.

Huerta-Solano, C.I. (2014). Enseñanza de vocablos aislados y en contexto mediante un método basado en las características de los participantes sordos. Tesis de Maestría. Universidad de Guadalajara.

Huerta-Solano, C. I., Varela, J., Figueroa-González, J. A., Delgado-González, M. y Rosas Montoya, C. (2016). Evaluación de la competencia lectora de vocablos y frases seleccionadas en alumnos sordos mexicanos de bachillerato. Revista Mexicana de Psicología Educativa (RMPE), enero-diciembre, 4(1):15-28.

Kantor, J. y Smith, N. $(1975,2015)$. La ciencia de la psicología: Un estudio interconductual. México: Universidad de Guadalajara. Krakow, R. A. y Hanson, V. L. (1985). Deaf Signers and Seri- 
al Recall in the Visual Modality: Memory of Signs, Fingerspelling, and Print. Memory and Cognition, 13: 265-272.

Kuhn, T. S. (1962, 2004). Prioridad de los paradigmas. En A. Contín (Ed.), La estructura de las revoluciones científicas (pp. 79-90). Buenos Aires, Argentina: Fondo de Cultura Económica.

Kuntze, M. (1998). Literacy and Deaf Children: The Language Question. Topics in Language Disorders, 18(4): 1-15.

LaSasso, C. y Davey, B. (1987). The Relationship between Lexical Knowledge Comprehension for Prelingually, Profoundly Hearing-impaired Students. Volta Review, 89: 211-220.

Lederberg, A. R. y Everhart, V. S. (2000). Conversations between Deaf Children and their Hearing Mothers: Pragmatic and Dialogic Characteristics. Journal of Deaf Studies and Deaf Education, 5: 303-322.

Luckner, J. L., Sebald, A. M., Cooney, J. y Muir, S. G. (2005, 2006). An Examination of the Evidence-based Literacy Research in Deaf Education. American Annals of the Deaf, 150: 443-456.

Mies, B. A. (1992). El léxico escrito en la comprensión lectora: estudio de un grupo de alumnos sordos profundos. Logopedia, fonoaudiología y audiología, XII, 4: 203-212.

Minskoff, E. (2005). Teaching Reading to Struggling Learners. Baltimore: Brookes.

Paul, P. y Gustafson, G. (1991). Hearing-impaired Students' Comprehension of High Frequency Multimeaning Words. Remedial and Special Education (RASE), 12: 52-62.

Paul, P. (1998). Literacy and Deafness: The Development of Reading, Writing, and Literature Thought. Boston, MA: Allyn y Bacon.

Paul, P. (2003). Processing and Components of Reading. In Marschark, M. y Spence, P. E. (Eds), Oxford HandBook of Deaf Studies, Language and Education (pp. 97-109). Oxford, England: Oxford University Press.

Paul, P., Wang, Y. y Williams, C. (2013). Deaf Students and the Qualitative Similarity Hypothesis: Understanding Language and Literacy Development. Washington, DC: Gallaudet University Press. 
Quigley, S., McAnally, P., King, C. y Rose, S. (1991). Reading Milestones: Teacher's Guide. Austin: TX: Pro-Ell.

Quigley, S., McAnally, P., Rose. S., Payne, J. A. y Paul, P. V. (2003). Reading Bridge: Mosaic. (2nd ed.). Austin. TX: Pro-Ed.

Ribes, E. y López, F. (1985). Teoría de la conducta. Un modelo de campo y paramétrico. México: Editorial Trillas.

Sánchez-López, P. y Rodríguez, R. (1997). Bilingüísmo: Bases para la intervención psicológica. Madrid: Síntesis.

Schirmer, B. R. y Williams, C. (2003). Approaches to Teaching Reading. En Marschark, M. y Spencer, P. E. (Eds.), Oxford Handbook of Deaf Studies, Language, and Education (pp. 110-122). Oxford, UK: Oxford University Press.

Schimmel, C. S., Edwards, S. G. y Prikett H. T. (1999). Reading?... Pah! (I Got It!): Innovative Reading Techniques for Successful Deaf Readers. American Annals of the Deaf, 144: 298-308.

Supalla, S. (1990). Segmentation of Manually Coded English: Problems with the Mapping of English in the Visual-gestural Mode. Doctoral Dissertation, University of Illinois, Champaign.

Tello-Miranda, O. A., Varela, J. y Palos-Toscano, U. (en dictamen). Revisión sistemática sobre los factores que influyen en el desarrollo de la competencia lectora de personas sordas.

Traxler, C. B. (2000). The Stanford Achievement Test, 9th Edition: National Norming and Performance Standards for Deaf and Hard of Hearing Students. Journal of Deaf Studies and Deaf Education, 5: 337-348.

Varela, J. (2008). Conceptos básicos del interconductismo: para entender el interconductismo. México: Universidad de Guadalajara.

Varela, J., Huerta-Solano, C. I., Nava, G. y Ortega, P. (2014). Entrenamiento lingüístico con personas sordas. En G. Nava (Coord.), Aplicaciones actuales en psicología educativa (pp. 165-192). Guadalajara, Jalisco: Editorial Universitaria. 